\title{
Influencia de la Estructura de los Parques Eólicos en el Costo y la Rentabilidad
}

\author{
Lorenzo A. Enríquez ${ }^{(1,3)}$, Luis. J. García(2), Carlos Miranda ${ }^{(3)}$ y Alex R. Jordán ${ }^{(3)}$ \\ (1) Facultad de Informática y Electrónica, Escuela superior Politécnica de Chimborazo, Riobamba, Ecuador \\ (e-mail: lenriquez@espoch.edu.ec) \\ (2) Centro de Estudios de Energía y Refrigeración, Fernando Brossard Pérez, Universidad de Oriente, Santiago de Cuba- \\ Cuba. (e-mail: Igarcia@uo.edu.cu) \\ (3) Centro de Energías Alternativas y Ambiente, Grupo de Energía Eólica, Escuela superior Politécnica de Chimborazo, \\ Riobamba-Ecuador (e-mail: c7miranda@gmail.com; arjv10@outlook.com)
}

Recibido Dic. 12, 2018; Aceptado Feb. 8, 2019; Versión final Mar. 28, 2019, Publicado Oct. 2019

\begin{abstract}
Resumen
En este trabajo se propone la solución de dos de los principales problemas que deben ser resueltos en las primeras etapas del proyecto de los parques eólicos, la estructura que deberá tener el parque y su rentabilidad. Se aplica un modelo matemático desarrollado a partir del método paramétrico que permite determinar de forma rápida y con aceptable precisión el costo capital mediante parámetros técnicos relevantes. El modelo fue calibrado mediante una base de datos representativa de los países de América Latina que mayor utilización hacen de la energía eólica. Se utilizó para definir la estructura más adecuada del proyecto de cinco parques en la provincia de Chimborazo, en Ecuador. Se concluye que el número de máquinas, sus potencias y alturas de las torres son los tres parámetros que determinan el costo del parque y al mismo tiempo, son los que definen la energía producida y la rentabilidad, por lo que un modelo de costo que integre esas variables resulta una importante herramienta cuando se aplican criterios de validación económicos-financieros como el Valor Presente Neto, el Costo nivelado de la Energía, el Costo del Ciclo de Vida y otros.
\end{abstract}

Palabras clave: estimación paramétrica; escalado del costo; índices de costo; rentabilidad

\section{Influence of Structure of Wind Farms on Cost and Profit}

\begin{abstract}
The solution for the two of the main problems that must be solved in the first stages of the wind farm project: the park structure and its profitability. A mathematical model developed from the parametric method is applied, which allows determining quickly and with acceptable precision the capital cost through relevant technical parameters. The model was calibrated through a database representative of the Latin American countries that make the most use of wind energy. Was applied to define the best appropriate structure of the project of five parks in the province of Chimborazo, Republic of Ecuador, reaching the conclusion that the number of machines, their powers and heights of the towers, are the three parameters that determine the cost of the park, and that at the same time, are those that define the energy produced and profitability, so a cost model that integrates these variables, is an important tool when applying economic-financial validation criteria such as Net Present Value, the specific cost of energy, the life cycle cost and others
\end{abstract}

Keywords: parametric estimation; economic scaling; cost index; profit 


\section{INTRODUCCION}

El costo total durante el período de vida útil del proyecto está compuesto por el costo de la inversión o costo capital y los costos de operación, mantenimiento y reemplazo de los equipos que tienen un tiempo de vida menor que la del proyecto y el costo de combustible en aquellos proyectos que lo consumen (Bueno et al, 2016). El costo remanente de los equipos e instalaciones que todavía pueden ser utilizados en otras actividades o vendidos como materia prima se resta del costo total del proyecto. El costo capital de los parques eólicos constituye la componente más compleja de calcular (Enríquez et al, 2016), en la práctica se puede llevar a cabo mediante dos procedimientos generales: la determinación paso a paso o discreta y la determinación mediante algún método de estimación "(Conde et al, 2015)".

El cálculo discreto del costo de los proyectos normalmente se realiza mediante la suma de los costos individuales de ingeniería, equipamientos, materiales y labor dentro del costo capital (Enríquez et al, 2018). Las partes pueden variar en tamaño y número desde unos pocos hasta cientos o miles de tareas o paquetes de trabajo discretos. Este cálculo generalmente es realizado por el contratista y viene acompañado por la programación de cada actividad (Fernández y Crespo, 2015). El resultado puede ser preciso si se dominan todos los factores determinantes del costo, pero es laborioso, consume mucho tiempo de trabajo y no revela la relación existente entre los parámetros técnicos y el costo. Existen diferentes métodos no discretos de estimación del costo capital. En general, pueden estar contenidos dentro de los siguientes cuatro grupos: 1) estimación por el costo relativo de la potencia; 2) estimación del costo por analogía; 3) estimación por la sumatoria del costo de los componentes; 4) estimación del costo por el elemento más representativo (Conde et al, 2015).

La estimación por el costo relativo de la potencia es el método más sencillo y uno de los más utilizados para una primera estimación. (Kogan y Bondorevsky, 2016). El costo de la turbina o del parque se estima multiplicando la potencia por el valor promedio de la relación $\$ / \mathrm{kW}$ obtenido estadísticamente. Las principales organizaciones de la energía eólica publican con frecuencia este índice para diferentes regiones del mundo (Taylor et al., 2015). Puede considerarse un modelo lineal de una sola variable: la potencia. Presenta las desventajas de que ignora las leyes del escalado económico y la influencia que ejerce la estructura del parque en el costo, definida por el número de máquinas, sus potencias y alturas de las torres (Giménez et al. ,2011). Al expresarse mediante el rango de valores $(1800 \$ / \mathrm{kW}-2400 \$ / \mathrm{kW})$ para América Latina, presenta un margen de error que puede llegar a $\pm 15 \%$

La estimación del costo por analogía utiliza como referencia la programación llevada a cabo para estimar el costo de un proyecto realizado con anterioridad (Manwell et al., 2009), se fundamenta en la comparación y extrapolación del costo de elementos y actividades similares; es útil durante los estudios de pre factibilidad económica cuando aún no se dispone de suficiente información; tiene una fuerte influencia la "opinión de experto; se considera un método discreto sintetizado que debe ejecutarse en cada ocasión.

Estimación por la sumatoria del costo de los componentes: el costo capital se calcula mediante la suma de los costos de los sistemas que conforman las turbinas (Burton et al., 2002) en función del peso de material consumido en cada uno de ellos mediante un coeficiente característico que depende del material y un factor que tiene en cuenta la complejidad del sistema. Los valores del coeficiente característico y el factor de complejidad para cada elemento se determinan de forma experimental. En otras ocasiones se toman otros elementos de las turbinas como el diámetro del rotor (Castro et al, 2008), El nivel de precisión que se logra es bajo, tampoco refleja la influencia de los parámetros relevantes en el costo.

Estimación del costo por el elemento más representativo: Este método tiene su fundamento en la fracción (\%) que representa cada elemento en el costo total. Se le denomina comúnmente método del pastel porque generalmente se utiliza ese tipo de gráfico para la distribución porcentual del costo de cada componente (Blanco, 2009), (Figueredo et al, 2017). En él se describe la distribución del costo de los componentes de los parques eólicos en Europa y Norte América, no incluyen los costos de transportación marítima, construcción de vías de acceso, alquileres de grúas y las tasas de interés son bajas; esas son algunas de las causas que ocasionan que el costo de los proyectos en los países que no producen el equipamiento y no presentan altos niveles de desarrollo sea considerablemente mayor. Esta herramienta es útil al igual que la anterior para la etapa de pre factibilidad y para establecer comparación cruzada con otros métodos.

Del análisis de los métodos anteriores se puede concluir que todos presentan las mismas deficiencias: 1) la relación entre los parámetros de estimación y el costo la establecen de forma lineal, ignoran las leyes del escalado económico (L. Blank et al., 2002), y del aprendizaje (Taylor y Llas., 2015); 2) no revelan la relación existente entre el costo y los parámetros relevantes del proyecto; 3) el método del elemento más representativo para el caso de la turbina fracciona esta en varias partes y establece la relación de cada una de ellas con el costo de forma lineal; si cada una de esas partes es proporcional a la potencia de la turbina, 
entonces surge la siguiente pregunta: ¿por qué no se toma la potencia como parámetro relevante del costo de la turbina? Igualmente ocurre con el fraccionamiento del parque; si todos los elementos del parque son proporcionales a la potencia del parque, ¿por qué no se toma la potencia a instalar como el parámetro relevante del costo del parque?

\section{MÉTODO UTILIZADO}

Para poder determinar la mejor estructura del parque, dada por el número de máquinas, sus potencias y alturas de las torres, fue necesario desarrollar un modelo de estimación del costo capital en el cual estuvieran involucrados esos tres parámetros. El método de estimación paramétrica supera las deficiencias que presentan el método discreto y los diferentes métodos de estimación no paramétricos. Consiste en una técnica que emplea una o más relaciones de las variables técnicas que no son utilizadas con este propósito, tales como: potencia, energía, flujo, torque, calor, empuje, tensión, resistencia, presión, temperatura, peso y muchas otras, con el costo del proyecto (Panai et al, 2015); desecha todos los procedimientos seguidos tradicionalmente; utiliza como ciencias auxiliares la Matemática, la Estadística y la Lógica (Eck et al, 2008), (Shokrzadeh, 2014). .Para el desarrollo del modelo se siguió el algoritmo mostrado en la figura 1.

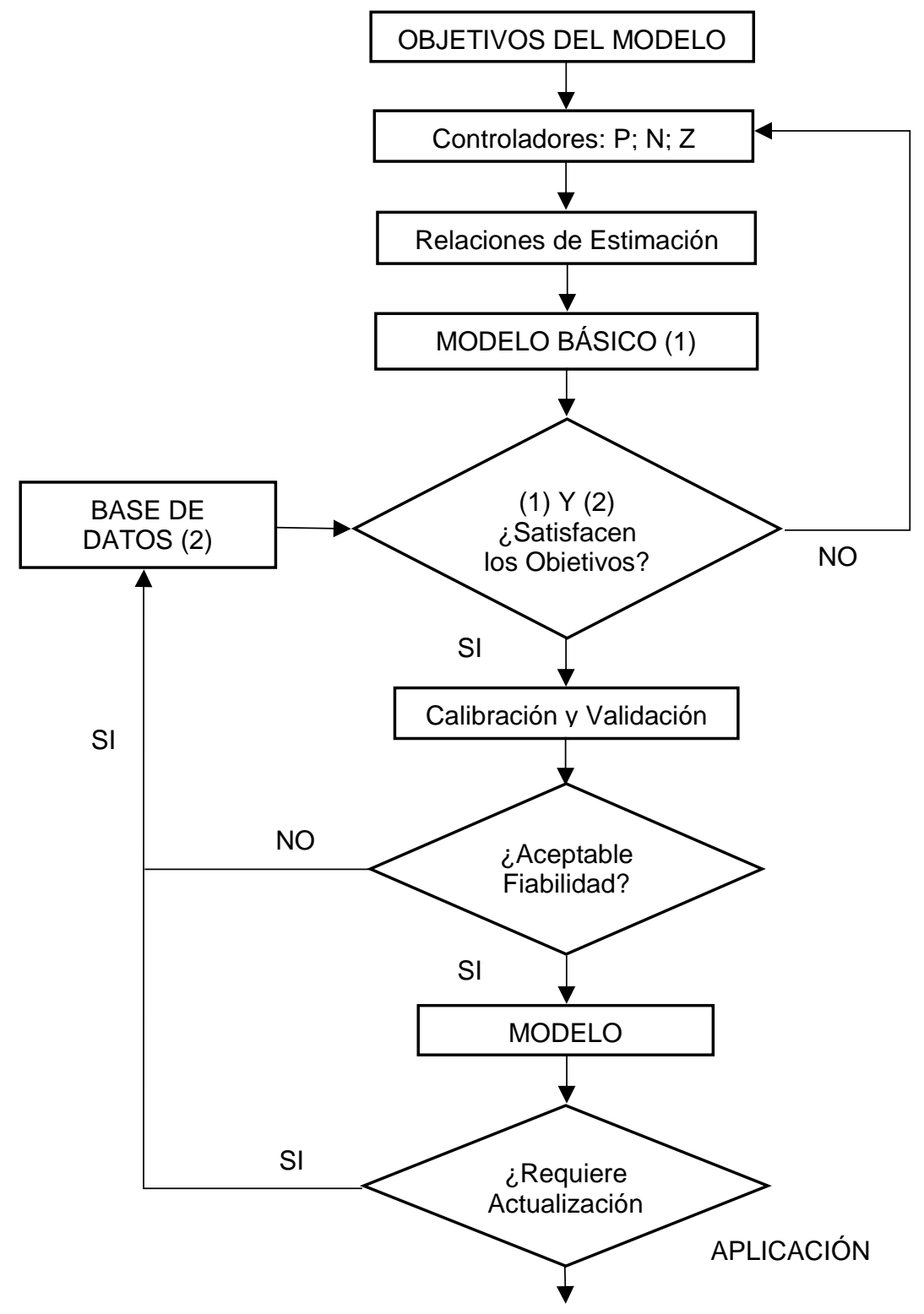

Fig. 1: Algoritmo diseñado para el desarrollo de la ecuación paramétrica de estimación del costo de los parques eólicos.

\section{Deducción de los parámetros controladores}

Es evidente que con el aumento de las dimensiones de los elementos y más específicamente del volumen aumenta proporcionalmente el material consumido y consecuentemente el costo; por otra parte, no es muy difícil demostrar mediante la teoría de los modelos que para máquinas semejantes la potencia de esta 
depende de sus dimensiones. Para el caso de las turbinas eólicas la potencia es proporcional al área del rotor, dada por la ecuación (1)

$$
P_{t}=\frac{1}{2} C_{P} \cdot \rho \cdot A \cdot v^{3} \cdot \eta_{t} \quad W
$$

Además, la potencia también determina el costo del equipamiento eléctrico, líneas de transmisión y hasta la transportación y montaje de las máquinas. Entonces, ¿por qué considerar el diámetro del rotor $u$ otra dimensión y no la potencia el parámetro relevante del costo de la turbina? A su vez, la potencia de las turbinas y su número determinan la potencia del parque y su costo, por lo tanto, un segundo parámetro relevante es el número de turbinas $(\mathrm{N})$.

Un tercer parámetro a considerar lo constituye la altura de la torre. Es de esperar que el aumento del costo con la altura se produzca de forma exponencial porque de esa misma manera se produce el aumento de la carga aerodinámica sobre la torre, el rotor, la góndola y otros elementos, lo cual impone mayores exigencias a los materiales y procesos constructivos; aumentan los costos de transportación y montaje y también con frecuencia las tasas de seguros.

Sin embargo, con el aumento de la altura se logra mayor captación de la energía del viento. Estos tres parámetros también definen la energía desarrollada por la turbina, lo cual facilita cualquier análisis tecnoeconómico del proyecto. Es necesario comprobar si son suficientes esos parámetros y la forma en que están vinculados para definir el costo del parque. (Enríquez et al, 2018). Se plantea la siguiente hipótesis: Es posible estimar el costo de los parques eólicos con aceptable precisión mediante una ecuación que relacione el costo con la potencia, el número y altura del buje de las turbinas.

\section{Desarrollo de la base de datos}

El costo capital de los parques eólicos difiere en las diferentes regiones del mundo. En América Latina, con excepción de Brasil, el costo del kW instalado es superior al de Europa, China y América del Norte (Pinilla, 2018), (IRENA, 2016). Sin embargo, por la información que se ha recopilado de aproximadamente 80 parques de América Latina, puestos en funcionamiento en el 2012, considerado año cero a los efectos de la ley de aprendizaje en el modelo, se ha podido comprobar que de los países que presentan mayor potencia instalada no existe una marcada diferencia en el costo promedio $(\$ / \mathrm{kW})$, aunque entre ellos existan diferencias apreciables de transportación marítima, lejanía de las redes de distribución, topografía, etc.

Esto indica que la influencia que representan esos factores no es representativa y están influenciados por la potencia instalada. Se puede establecer una segunda hipótesis: Es posible estimar el costo de los parques de los países seleccionados de América Latina con aceptable precisión mediante una ecuación paramétrica desarrollada con la base de datos que tiene como información la potencia de las turbinas, su número, alturas de las torres y el costo capital de esos parques.

\section{Formulación del modelo matemático de estimación paramétrica del costo}

El modelo desarrollado se fundamenta en las dos leyes de la economía indicadas anteriormente (Taylor y Llas, 2015) y (Eck et al, 2008), por lo que el costo debe ser una función no lineal de los parámetros relevantes. Se propone un modelo compuesto por el producto de factores exponenciales, el cual contempla todas las posibilidades: relaciones crecientes, decrecientes, negativas e inclusive lineales, en dependencia de los valores que tomen los exponentes. La relación entre el costo capital del parque que se proyecta (con subíndices 2) y un parque modelo, construido en condiciones normales (con subíndices 1), se puede expresar mediante la ecuación (2)

$$
\frac{\mathrm{C}_{\mathrm{P}_{2}}}{\mathrm{C}_{\mathrm{P}_{1}}}=\sum_{\mathrm{r}=1}^{\mathrm{r}=\mathrm{x}}(\mathrm{REC})^{\mathrm{x}} \cdot \prod_{\mathrm{l}=0}^{\mathrm{l}} \mathrm{I}_{\mathrm{C}_{1}}
$$

Teniendo en cuenta las relaciones de estimación del costo para los tres parámetros relevantes, se puede plantear con la ecuación (3).

$$
\frac{\mathrm{C}_{\mathrm{P}_{2}}}{\mathrm{C}_{\mathrm{P}_{1}}}=\left(\frac{\mathrm{P}_{\mathrm{P}_{2}}}{\mathrm{P}_{\mathrm{P}_{1}}}\right)^{\mathrm{a}} \cdot\left(\frac{\mathrm{N}_{2}}{\mathrm{~N}_{1}}\right)^{\mathrm{b}} \cdot\left(\frac{\mathrm{Z}_{2}}{\mathrm{Z}_{1}}\right)^{\mathrm{d}} \cdot \prod_{\mathrm{l}=0}^{\mathrm{l}} \mathrm{I}_{\mathrm{C}_{\mathrm{l}}}
$$


Se aplican índices de costos cuando existen condiciones anormales debido a extensas carreteras, grandes elevaciones de difícil acceso, condiciones climáticas adversas dadas por velocidades extremas del aire, turbulencia y otras que pueden encarecer los costos de los proyectos. Aquellos parques que no presentan esas condiciones se consideran normales y los índices de costo se toman igual a la unidad. El costo de los parques que están dentro del entorno promedio de la inmensa mayoría de los parques se considera normal. En ese caso, la ecuación (3) se puede expresar mediante la ecuación (4).

$$
\frac{\mathrm{C}_{\mathrm{P}_{2}}}{\mathrm{C}_{\mathrm{P}_{1}}}=\left(\frac{\mathrm{P}_{\mathrm{P}_{2}}}{\mathrm{P}_{\mathrm{P}_{1}}}\right)^{a} \cdot\left(\frac{\mathrm{N}_{2}}{\mathrm{~N}_{1}}\right)^{b} \cdot\left(\frac{\mathrm{Z}_{2}}{\mathrm{Z}_{1}}\right)^{d}
$$

También se puede aplicar un índice de costo que tiene en cuenta las leyes de aprendizaje. En la investigación se demuestra que el costo de los parques sigue una tendencia decreciente hasta el 2030 (Rubin et al., 2015), la cual está dada por la ecuación (5).

$$
\mathrm{I}_{\mathrm{t}}=\mathrm{t}^{-0,05}
$$

\section{Modelo teórico básico}

Se puede proponer un modelo teórico básico para aquellos parques de América Latina cuyos costos están dentro de los límites promedio normales mediante la ecuación (6)

$$
\mathrm{C}=\mathrm{A} \cdot \mathrm{P}^{\mathrm{a}} \cdot \mathrm{N}^{\mathrm{b}} \cdot \mathrm{Z}^{\mathrm{d}} \quad \$
$$

El valor del coeficiente A se puede obtener de la ecuación (5) con los valores conocidos de los parámetros relevantes del parque modelo $\left(P_{P_{1}} N_{1}\right.$ y $\left.Z_{1}\right)$ y el costo $C_{P 1}$ para condiciones normales (Ic=1), el cual se expresa en la ecuación (7)

$$
A=C_{P 1} \cdot\left(\frac{1}{P_{P 1}}\right)^{a} \cdot\left(\frac{1}{N_{1}}\right)^{b} \cdot\left(\frac{1}{Z 1}\right)^{d}
$$

Se demuestra analíticamente (Enríquez et al., 2016), que para máquinas de igual altura $(Z 1=Z 2)$ y poniendo la ecuación (4) en función de la potencia del parque:

$$
\left(\frac{C_{P 2}}{C_{P 1}}\right)=\left(\frac{P_{P 2}}{P_{P 1}}\right)^{a} \cdot\left(\frac{N_{2}}{N_{1}}\right)^{b}
$$

Si se expresa en función de la potencia de las turbinas:

$$
\begin{aligned}
& \left(\frac{C_{P 2}}{C_{P 1}}\right)=\left(\frac{P_{T 2} \cdot N_{2}}{P_{T 1} \cdot N_{1}}\right)^{a} \cdot\left(\frac{N_{2}}{N_{1}}\right)^{b}=\left(\frac{P_{T 2}}{P_{T 1}}\right)^{a} \cdot\left(\frac{N_{2}}{N_{1}}\right)^{(a+b)} \\
& \left(\frac{C_{P 2}}{C_{P 1}}\right)=\left(\frac{P_{T 2}}{P_{T 1}}\right)^{a} \cdot\left(\frac{N_{2}}{N_{1}}\right)^{c}
\end{aligned}
$$

Donde:

$P_{\mathrm{P} 1}$ y $\mathrm{P}_{\mathrm{P} 2}$ - Potencias de los parques 1 y 2 respectivamente $\mathrm{P}_{\mathrm{T} 1}$ y $\mathrm{P}_{\mathrm{T} 2}$ - Potencias de las turbinas 1 y 2 respectivamente

Como las ecuaciones (8) y (9) tienen que arrojar el mismo resultado, se tiene que: $c=a+b$. En muchos proyectos, para simplificar, se toma incorrectamente el valor de $c=1$, considerando lineal el costo con el número de máquinas, en ese caso:

$a+b=1$

Si se recurre a la teoría de las seis décimas, dándole al exponente de la potencia $a=0,6$; se obtiene $b=0,4$, y c=1. 
El aumento de la altura de la torre ha estado acompañado por el aumento de potencia de las máquinas, por lo que la presencia del exponente $d$ modifica los valores de los exponentes a y b, por tanto, el coeficiente $A$ y los exponentes $\mathrm{a}$, b y d, solo pueden ser determinados mediante procedimientos estadísticos.

\section{Ajuste y validación del modelo simplificado}

El ajuste del modelo simplificado, ecuación (6) fue realizado con los parámetros de 23 parques de 11 países latinoamericanos y caribeños tomados al azar de forma proporcional a las potencias instaladas, puestos en funcionamiento en el 2012, considerado año cero para los efectos de la ley de aprendizaje. En la tabla 1 se presentan los parques seleccionados. Utilizando la base de datos, aplicando logaritmos a ambos miembros de la ecuación (6) para linearizarla y mediante la función estimación lineal de EXCEL se obtuvieron el coeficiente A, los exponentes a, b y d y la matriz con la estadística de la ecuación. Luego las ecuaciones (9), en función de la potencia del parque y (10), en función de la potencia de las máquinas fueron comprobadas mediante la herramienta Statgraphics.

En función de la potencia del parque:

$$
\mathrm{C}=18,02 \cdot \mathrm{P}_{\mathrm{P}}^{0,675} \cdot \mathrm{N}^{0,249} \cdot \mathrm{Z}^{1,73} \$
$$

En función de la potencia de los aerogeneradores:

$$
\mathrm{C}=18,02 \cdot \mathrm{P}_{\mathrm{t}}^{0,675} \cdot \mathrm{N}^{0,924} \cdot \mathrm{Z}^{1,73}
$$

Puede comprobarse la relación entre los componentes a, b y c dada anteriormente

Tabla 1: Base de datos de los parques seleccionados

\begin{tabular}{|l|l|c|c|c|c|}
\hline \multicolumn{1}{|c|}{ País } & \multicolumn{1}{|c|}{$\begin{array}{c}\text { Nombre del } \\
\text { parque }\end{array}$} & $\begin{array}{c}\text { Potencia } \\
\text { Nominal }(M W)\end{array}$ & $\begin{array}{c}\text { Costo real } \\
X 10^{6} \$\end{array}$ & $\begin{array}{c}\text { Costo calculado } \\
X 10^{6} \$\end{array}$ & Error (\%) \\
\hline Argentina & Malas pina & 50,0 & 118,0 & 115,4 & 2,2 \\
\hline Chile & Arrayan & 115,0 & 245,0 & 240,0 & 2,0 \\
\hline Chile & Totoral & 46,0 & 140,0 & 131,0 & 6,4 \\
\hline Chile & El pacífico & 70,0 & 144,0 & 157,0 & $-9,2$ \\
\hline Chile & Punta Colorada & 20,0 & 50,0 & 49,7 & 0.6 \\
\hline Chile & Las Peñas & 9,0 & 20,0 & 19,4 & 3,0 \\
\hline Colombia & Jepirachi & 19,5 & 33,1 & 32,8 & 0.6 \\
\hline Costa Rica & Chiripa & 49,5 & 125,0 & 122,7 & 1.8 \\
\hline Cuba & Gibara II & 4,5 & 7,7 & 7,7 & 0,0 \\
\hline México & El Porvenir & 54,0 & 140,0 & 127,1 & 9,2 \\
\hline México & La Ventosa & 250,5 & 550,0 & 545,5 & 0,8 \\
\hline México & Dos Arbolitos & 70,0 & 200,0 & 192,9 & 3,6 \\
\hline México & Pier II & 66,0 & 150,0 & 149,9 & 0,1 \\
\hline México & Dominica & 100,0 & 225,0 & 218,4 & 2,9 \\
\hline México & Dominica II & 100,0 & 225,0 & 218,4 & 2.9 \\
\hline Panamá & Penonomé & 220,0 & 570,0 & 523,8 & 8,9 \\
\hline Perú & Tres Hermanas & 90,0 & 185,0 & 168,4 & 9,0 \\
\hline Perú & Cupisnique & 81,0 & 234,0 & 243,0 & $-3,9$ \\
\hline Puerto Rico & Santa Isabel & 101,2 & 200,0 & 213,4 & $-6,7$ \\
\hline R. Dominicana & Los Cocos & 77,2 & 200,0 & 212,9 & $-6,5$ \\
\hline Uruguay & Melowind & 50,0 & 100,0 & 97,8 & 2,2 \\
\hline & & & & Error promedio & 1,2 \\
\hline
\end{tabular}


El resultado que se obtiene con ambas ecuaciones es el mismo. Estas fueron evaluadas en los siguientes rangos: potencia del parque desde 4,5 MW hasta $250 \mathrm{MW}$; número de turbinas desde 5 hasta 167; altura de las torres desde $52 \mathrm{~m}$ hasta $90 \mathrm{~m}$. La evaluación estadística de ambas ecuaciones demuestra que:1) Los tres parámetros seleccionados son excelentes estimadores del costo de los parques $\left.\left(r^{2}>0,98\right) ; 2\right)$ El estimador de Fisher calculado $(1755,56)$ es mucho mayor que el crítico $(5,01)$ para $\alpha=0,99$ lo que demuestra que esa relación no ha sido casual 3) El error estándar de las variables fue: $\mathrm{SeP}=0,081(8,1 \%) ; \mathrm{SeN}=0,02(2 \%)$; $\mathrm{SeN}=0,18(18 \%)$ y de la variable dependiente $\mathrm{SeC}=0,062(6,2 \%)$

\section{Tamaño de la muestra}

A partir de la ecuación semi-empírica dada por la expresión (13) (Morales et al., 2012)

$$
\mathrm{n}=\frac{Z_{0}^{2} \cdot \mathrm{N} \cdot \sigma^{2}}{\mathrm{e}^{\star 2}(\mathrm{~N}-1)+Z_{0}^{2}+\sigma^{2}}
$$

Con $\mathrm{N}=78 ; \mathrm{e}^{*}=0,0438(4,38 \%) ; \mathrm{Z}_{0}=2,58$ (99\% de nivel de confianza), el cual se determina en la tabla de distribución normal estándar y $\sigma^{2}=0,062(6,2 \%)$, se obtuvo un tamaño de muestra $n=11,52$ es decir, hubiera sido suficiente una muestra de 12 parques, por lo que con la muestra tomada de 23 se tiene un nivel de confianza de los resultados mayor del esperado.

Los resultados anteriores permiten afirmar que el modelo matemático propuesto es una buena representación de la ecuación para la estimación paramétrica del costo capital de los parques eólicos en las condiciones indicadas; por tanto, se puede concluir que las hipótesis propuestas para estimar con suficiente precisión el costo capital de las turbinas y de los parques eólicos de los países que integran la base de datos de América Latina y el Caribe, son verdaderas.

\section{RESULTADOS Y DISCUSIÓN}

Con la aplicación del modelo paramétrico se cumplen dos de los objetivos fundamentales del proyecto de los parques eólicos: la determinación de la mejor estructura del parque y los beneficios que éste puede aportar. Los resultados que se exponen a continuación fueron llevados a cabo durante los estudios de factibilidad del parque Rumignokiana-Cañi en la provincia de Chimborazo, República de Ecuador, donde por las condiciones topográficas adversas y disminución de la densidad del aire con la altura, resulta imprescindible optimizar los proyectos para lograr mayores beneficios técnicos y económicos (Van et al., 2016).

\section{Influencia de la estructura del parque en el costo}

Para una potencia de proyecto del parque de $24 \mathrm{MW}$ fueron analizados los costos con máquinas de diferentes potencias: $800 \mathrm{~kW}, 1500 \mathrm{~kW}$ y $2000 \mathrm{~kW}$, con 30, 16 y 12 aerogeneradores respectivamente y dos niveles de alturas, $65 \mathrm{~m}$ y $78 \mathrm{~m}$, lo cual hace un total de seis alternativas. Al aplicar la ecuación (12) se obtienen los resultados que muestra la figura 2.

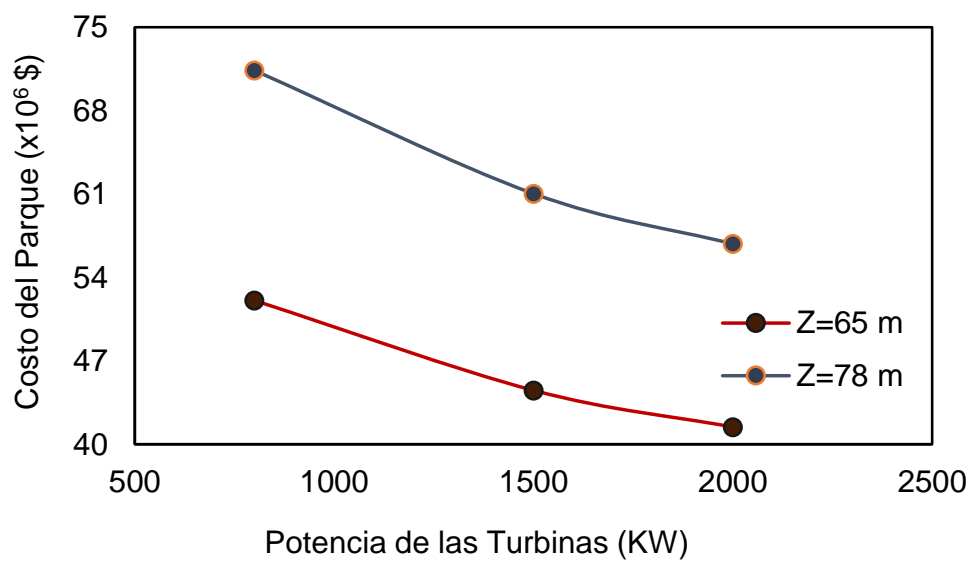

Fig. 2: de la potencia, número y alturas de las máquinas en el Costo del Parque.

En la figura 2 se puede observar que la peor opción desde el punto de vista del costo es con el mayor número de máquinas y mayor altura. Para una misma altura a medida que aumenta la potencia de las máquinas y disminuye el número de ellas, el costo se hace menor. Mediante otros métodos de estimación de costo sería imposible realizar este análisis. (Ávila et al, 2010) hacen un estudio similar mediante el método del costo 
relativo de la potencia para cuatro parques de aproximadamente $12 \mathrm{MW}$ con 15 turbinas de $800 \mathrm{~kW}, 13$ de $900 \mathrm{~kW}$, seis de $2000 \mathrm{~kW}$ y cinco de $2300 \mathrm{~kW}$, todas con la misma altura de $78 \mathrm{~m}$. Consideran el mismo costo relativo de las turbinas de $1400 \$ / \mathrm{kW}$. No tienen en cuenta la influencia de la potencia, el número de máquinas y la altura en el costo; el costo relativo tomado corresponde en Cuba a máquinas cuya altura está por debajo de $50 \mathrm{~m}$. Por las razones anteriores, el costo de los parques les resulta aproximadamente el mismo con las cuatro variantes y el criterio de validación financiera escogido no es confiable pues está permeado de errores.

\section{Influencia de la estructura en la rentabilidad del parque}

El costo del parque, de por sí solo no define cuál es la mejor variante, para ello debe aplicarse un método de validación financiera, preferiblemente de aquellos que utilizan la actualización del dinero en el tiempo; uno de los más generalizados es el Valor Presente Neto (VPN) del proyecto, que en su forma más sencilla está dado por la ecuación (14) (Pinzón et al, 2015) y (Herrera et al, 2011). Tiene la ventaja de que en una misma ecuación se relacionan el costo capital, dado en función de los parámetros técnicos, con los flujos de caja o de activos (Ingresos y egresos). En el caso de los parques eólicos, los ingresos son el resultado de la venta de la electricidad producida (valor +) y los egresos son los atribuidos a operación, mantenimiento y otros gastos en los que se incurre anualmente (valor -), es decir:

$$
V P N=-C+V_{r}+\sum_{n=1}^{n} \frac{F C_{n}}{(1+i)^{n}} \quad \$
$$

La energía captada por las máquinas en un tiempo dado generalmente en el año, se puede determinar por la potencia desarrollada para cada velocidad del viento $\mathrm{P}\left(\mathrm{v}_{\mathrm{i}}\right)$ en el intervalo de operación de la turbina desde la velocidad de arranque $v_{a}$ hasta la de corte $v_{c}$ y la probabilidad de ocurrencia de cada velocidad del viento durante el año $p(v i)$. Está dada por la ecuación (15).

$$
E=8760 \sum_{v_{a}}^{v_{c}} P\left(v_{i}\right) \cdot p\left(v_{i}\right) \quad k W h / a
$$

La probabilidad de ocurrencia de cada velocidad del viento se puede representar con suficiente precisión mediante la función de los parámetros de forma $\mathrm{k}$ y de escala $\mathrm{c}$ de Weibull (Junaid et al, 2015). Para el proyecto de Rumignokiana-Cañi en la provincia de Chimborazo, República de Ecuador, los parámetros del aire medidos a la altura de la torre de $65 \mathrm{~m}$ fueron: $\mathrm{k}=1,6 ; c=8,8 \mathrm{~m} / \mathrm{s}$ y la densidad del aire $\rho=0,87 \mathrm{~kg} / \mathrm{m}^{3}$; para altura de $78 \mathrm{~m}$, la densidad del aire y $\mathrm{k}$ mantienen su valor, pero el parámetro de escala $\mathrm{c}=9 \mathrm{~m} / \mathrm{s}$ ]. Con valores de probabilidad de velocidades del viento $p(v i)$ y de potencia $P(v i)$ en el rango de utilización de las turbinas seleccionadas, y aplicando la ecuación (15), se obtiene la energía generada en el año y con el cálculo de los restantes parámetros (costos de operación y mantenimiento, tasa de descuento anual, tarifa de venta de la electricidad, eficiencia del parque y otros), para todas las variantes se puede determinar la opción más rentable mediante el criterio financiero seleccionado. En la tabla 2, se resumen los resultados para el anteproyecto Rumignokiana-Cañi.

Tabla 2: Resumen del análisis económico-financiero

\begin{tabular}{|c|c|c|c|c|}
\hline \multirow{2}{*}{ Altura del buje } & & \multicolumn{3}{|c|}{ Tipo de Aerogeneradores } \\
\cline { 3 - 5 } & & $E-48-800$ & GW-70-1500 & G-87 \\
\hline \multirow{3}{*}{$65 \mathrm{~m}$} & Costo capital (\$) & 51360848 & 44029816 & 41033341 \\
\cline { 2 - 5 } & Energía $(G W h / a)$ & 53,90 & 55,56 & 59,29 \\
\cline { 2 - 5 } & VPN (\$) & 11180000 & 20470000 & 27830000 \\
\hline \multirow{3}{*}{$78 \mathrm{~m}$} & Costo capital (\$) & 70.406 .985 & 60.357 .387 & 56.249 .730 \\
\cline { 2 - 5 } & Energía (GWh/a) & 56,26 & 57,88 & 61,61 \\
\cline { 2 - 5 } & VPN (\$) & -5222000 & 6762000 & 15243000 \\
\hline
\end{tabular}

Observe, que la mayor rentabilidad (VPN=27,83 $10^{6} \$$ ) se obtiene para las máquinas de mayor potencia pero con altura de las torres de $65 \mathrm{~m}$ porque a pesar de haber un incremento de la energía para $78 \mathrm{~m}$ de altura, esta no es suficiente para compensar el aumento del costo del parque en ese emplazamiento. Esto es una demostración de que el costo capital del proyecto depende de la estructura que adopte el parque, dada por la combinación del número de máquinas, sus potencias y alturas de las torres; pero además, para determinar la mejor variante, debe completarse el estudio mediante un criterio de validación económico-financiera. Esto puede explicar, en parte, por qué en un mismo país con semejantes condiciones tecnológicas puede haber notables diferencias en el costo de parques de igual potencia instalada 


\section{CONCLUSIONES}

De acuerdo a los resultados de este estudio se pueden extraer las siguientes conclusiones principales:1) El método paramétrico permite determinar con aceptable precisión el costo y la rentabilidad de los parques eólicos. (2) El modelo propuesto fue aplicado a los estudios de seis micro localizaciones en la provincia de Chimborazo, República de Ecuador; se definió la mejor estructura de los parques pero debido a las grandes elevaciones con difícil acceso, fue necesario aplicar el criterio de condiciones extremas previsto en la ecuación mediante un índice de costo por analogía con el parque Villonaco, con altitud de $2730 \mathrm{~m}$, en la provincia de Loja, elevándose el costo de los proyectos en $30 \%(\mathrm{IC}=1,3)$; 3$)$ Al establecer el vínculo entre los parámetros técnicos y los económicos, se pudo realizar el estudio integral de factibilidad de las micro localizaciones; se comprobó que solo dos de los proyectos: Chimborazo, con densidad de potencia del viento de $250 \mathrm{~W} / \mathrm{m}^{2}$ y Rumignokiana-Cañi con $400 \mathrm{~W} / \mathrm{m}^{2}$ presentan rentabilidad financiera.

\section{NOTACIÓN}

\section{Símbolos}

$\begin{array}{ll}\mathrm{A} & \text { Área del rotor del aerogenerador }\left(\mathrm{m}^{2}\right) \\ \mathrm{a} & \text { Exponente de la potencia en la ecuación paramétrica } \\ \mathrm{b} & \text { Exponente del número de máquinas en la ecuación paramétrica } \\ \mathrm{C} & \text { Costo capital del parque }(\$) \\ \mathrm{C}_{\mathrm{P}} & \text { Coeficiente de potencia } \\ \mathrm{d} & \text { Exponente de la altura en la ecuación paramétrica } \\ \mathrm{E} & \text { Energía captada por la turbina } \mathrm{kWh} / \mathrm{a} \\ \mathrm{e}^{\star} & \text { Error medio de la ecuación paramétrica del costo }(\%) \\ \mathrm{l}_{\mathrm{t}} & \text { Îndice de costo (menor que uno) por la ley de aprendizaje } \\ \mathrm{i} & \text { Tasa de interés anual }(\%) \\ \mathrm{N} & \text { Tamaño de la población evaluada } \\ \mathrm{n} & \text { Tamaño de la muestra } \\ \mathrm{P}_{\mathrm{p}} & \text { Potencia del parque } \\ \mathrm{P}_{\mathrm{t}} & \text { Potencia de la turbina } \\ \mathrm{P}\left(\mathrm{v}_{\mathrm{i}}\right) & \text { Potencia de la turbina en función de la velocidad del viento }(\mathrm{kW}) \\ \mathrm{p}\left(\mathrm{v}_{i}\right) & \text { Probabilidad de ocurrencia de cada velocidad del viento }(\%) \\ \mathrm{V}_{\mathrm{r}} & \text { Valor residual del proyecto }(\$) \\ \mathrm{V} & \text { Velocidad del viento }(\mathrm{m} / \mathrm{s}) \\ \mathrm{Z} & \text { Altura del buje de la turbina }(\mathrm{m})\end{array}$

\section{Abreviaciones}

$\begin{array}{ll}\text { LCOE } & \text { Costo nivelado de la energía }(\$ / \mathrm{kWh}) \\ \text { FC } & \text { Flujo de caja }(\$) \\ \text { LCC } & \text { Costo del ciclo de vida del proyecto }(\$) \\ \text { VPN } & \text { Valor presente neto }(\$)\end{array}$

\section{Letras Griegas}

$\begin{array}{ll}\eta_{\mathrm{t}} & \text { Eficiencia total de la turbina } \\ \rho & \text { Densidad del aire }\left(\mathrm{kg} / \mathrm{m}^{3}\right) \\ \sigma & \text { Desviación estándar }\end{array}$

\section{REFERENCIAS}

Ávila, D., R. Prats, F. .García, Coste del kWh eólico generado en Cuba a partir de datos de viento de una región de buenos potenciales eólicos, Ingeniería Mecánica, ISSN: 1815-5944, 13(3), 38-45 (2010)

Blanco, M.I., The economics of wind energy. Department of Economics, University of Alcala Elsevier, Renewable and Sustainable Energy Reviews, 13, 1372-1382 (2009)

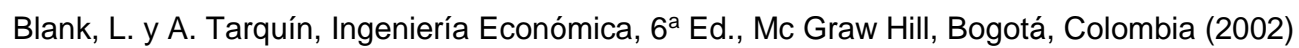

Bueno L., S.L. Rodríguez y S.P. Rodríguez, Análisis de costos de generación de energía eléctrica mediante fuentes renovables en el sistema eléctrico colombiano, doi:10.14482/inde.33.2.6368, Rev. Ingeniería y desarrollo, 34(2) 397419(2016)

Burton, T., D. Sharpe, N. Jenkins y E. Bossanyi, Wind Energy Handbook, ISBN: 0-471-48997-2, Wiley and Sons, 1(1), 630-635, England (2002) 
Castro, J. y M. Burgos, Optimización Global de Parques Eólicos Mediante Algoritmos Evolutivos, Tesis Doctoral, Universidad de Sevilla (2008)

Cerda A. y F. Vasquez, Differences Between Parametric and Non Parametric Estimation of Welfar Measures An Application to the Río Claro, Talca, Chile, ISSN:0716-1921, Rev. Redalyc, 23(31), 22-31(2005)

Conde A. y otros cincuenta y siete autores, Aeronautic and Space Administration (NASA), Cost Estimating Handbook, NASA, 4(1), (2015)

Eck, D., J. C. Smith y otros veinte y tres autores, Parametric Estimating Handbook, ISBN:0-9720204-7-0, Rev. International Society of Parametric analysts, 1(4),(2008)

Enríquez, L.A, L. García y C. Oro, Algorithm for The Techno-Economic Optimization Applied in Projects Of Wind Parks of Latin America, IOSR Journal of Mechanical and Civil Engineering, 13(4), 60-55 (2016)

Enríquez, L.A. y L. García, Parámetros controladores del costo capital de los parques eólicos, ISSN: 1815-5901, Rev. Ingeniería Energética, 39(1), 48-55 (2018)

Espejo, M.C. y M.R. García, La energía eólica en la producción de electricidad en España, doi: 10.4067/S071834022012000100007, Rev. Geografía norte grande, 51 (1),115-136(2012)

Fernandez, C.I., F. Puime y M.A. Crespo, Building up a model of cash-flow determination for a wind power project, ISSNe: 2174-3835, Rev. Atlantic Review of Economics, 1(1), 1-28 (2015)

Giménez, A.J. y J .C Gómez, Generación eólica empleando distintos tipos de generadores considerando su impacto en el sistema de potencia, ISSN: 2346-2183, Rev. Dyna, 78(169), 95-104(2011)

Herrera, O.S., S.A. Sarmiento, F.V. González y W. Schilling, Modelo de optimización de parques eólicos, ISSN: 18155944, Rev. Ingeniería Mecánica, 14(3),189-198(2011)

IRENA, Renewable Energy Technologies: Costs Analysis Series, Wind Power, Volume IRENA (2016)

Junaid, K. y otros seis, An Analysis of Wind Speed Distribution and Comparison of Five Numerical Methods for Estimating Weibull Parameters, ISSN: 2286-4822, Rev. European Academic Research, 2(1), 14007-14015 (2015)

Kogan, J. y D. Bondorevsky, La infraestructura en el desarrollo de América Latina, ISSN: 0252-8584, Rev. Economía y desarrollo, 150(1), 168-186(2016)

Manwell, J.F., J.G. Mc Gowan y A.L. Rogers, Wind Energy Explained. Theory, Design and Application Handbook, ISBN: 978-0-470-01500-1, Wiley and Sons, 1(2), England (2010)

Morales, P.V., Tamaño necesario de la muestra: ¿Cuántos sujetos necesitamos? (2012)

Panai, D., S. Deilami y M. Masoum, Evaluation of Parametric and Non-Parametric Methods for Power Curve Modelling of Wind Turbines, International Conference on Electrical and Electronics Engineering (ELECO), 996-1000, Bursa, Turquía, 26-28 de noviembre (2015)

Pinilla, A., El poder del viento, ISSN: 0121-4993, Rev. Ingeniería, 28(1) ,64-69(2008)

Rubin, E.S., M.L. Azevedo, P. Jaramillo y S. Yeh, A review of learning rate for electricity supply technologies, Rev. Elsevier, 86(1),198-218(2015)

Sergio, J.H. y G. León, Energía eólica en el istmo de Tehuantepec: desarrollo, actores y oposición social, ISSN: 03017036, Rev. Problemas del Desarrollo, 45(178), 139-162 (2014)

Shokrzadeh, S., M.J. Jozani y E. Bibeau, Wind Turbine Power Curve Modeling Using Advanced Parametrics and Non parametric Methods, doi: 10.1109/TSTE.2014.2345059, IEEE Trans actions on Sustainable Energy, 5(4), 1262-1269 (2014)

Taylor, M., K. Daniel, A. Llas y E. Young, Renewable Energy Generation Cost in 2014, ISBN: 978-92-95111-53-0, Rev. International Renewable Energy Agency (IRENA), 1(1), (2015)

Torres, D.A. y F.C Moreno, Evaluación del potencial eólico en el Consejo Popular de Cojímar, ISNN: 0864-151X, Rev. Cubana de Meteorología, 24(3), 256-267(2018)

Wiser, R, M. Hand, J. Seel y B. Paulos, Reducing Wind Energy Costs through Increased Turbine Size: Is the Sky the Limit? Rev. Berkeley National Laboratory Electricity Markets and Policy Group (2016) 